\title{
41. CICERBITA MURALIS SUBSP. GADITANA MEJÍAS (ASTERACEAE, CICHORIEAE), NUEVO TAXÓN ENDÉMICO DE LA PENÍNSULA IBÉRICA
}

\author{
José Antonio MEJÍAS
}

Recibido el 21 de octubre de 2015, aceptado para su publicación el 4 de noviembre de 2015

Cicerbita muralis subsp. gaditana Mejías (Asteraceae, Cichorieae), a new endemic taxa from the Iberian Peninsula

Palabras clave. Cádiz, Cicerbita, morfología del aquenio, Lactuca, Mycelis, Sierra de Grazalema.

Key words. Cadiz province, Cicerbita, achene morphology, Lactuca, Mycelis, Sierra de Grazalema.

Cicerbita muralis (L.) Wallr. [三 Lactuca muralis (L.) Gaernt., $\equiv$ Mycelis muralis (L.) Dumort.] es una especie propia de la Europa templada y áreas limítrofes (Feráková 1976, Preston \& Hill 1997), que alcanza por el norte la Península Escandinava, por el este el Cáucaso y las estribaciones de los montes Urales, y por el sur el norte de Marruecos y Argelia (Hultén \& Fries 1986). Vive en ambientes húmedos relativamente sombríos, sobre sustratos ricos en bases, frecuentemente calizos, aunque puede aparecer en otros tipos de suelos con distintos valores de pH (Czarnecka 1986, Clabby \& Osborne 1999). Sus poblaciones actuales parecen tener origen en un proceso post-glacial de recolonización desde regiones del sur del continente (Chauvet et al. 2004). En la Península Ibérica es frecuente en el norte, especialmente en la cordillera Cantábrica y Pirineos, y se hace progresivamente más rara hacia el sur, donde aparece muy localizada, prosperando sobre todo en roquedos, fondos de barrancos y grietas de zonas kársticas.

Pertenece a la subtribu Lactucinae de la tribu de las Cichorieae (Kilian et al. 2009), en la que parece ocupar una posición filogenética relativamente basal (Koopman 2005, Koopman et al. 1998, 2001). Dado que la historia evolutiva de este grupo es mal conocida (Wang et al. 2013) y que la circunscripción de sus géneros no está bien resuelta (Koopman et al. 1998, Kilian et al. loc.cit.), los criterios de clasificación han sido muy variables entre autores. Así, la especie ha sido incluida tanto en el género Lactuca L. (p. ej., Stebbins 1937) como en Cicerbita Wallr. (p. ej., Devesa \& Talavera 2014), si bien con mayor frecuencia lo ha sido en Mycelis Cass. (p. e, Feráková 1976; Bremer 1994), género que solo integraría esta especie. En la disparidad del tratamiento subyace una singular combinación de caracteres en la especie, de importancia taxonómica para la definición y clasificación de taxones en la subtribu, como son: las características del vilano, considerado dimórfico; la organización del involucro en dos series de brácteas claramente dispares, y la presencia de un pico bien definido en el fruto, como sucede en el género Lactuca, pero inusualmente corto $(1 / 3-1 / 7$ la longitud del cuerpo del aquenio; fig. 1B).

Este trabajo se ha desarrollado en el marco del proyecto Flora Iberica IX(2) del Plan Nacional de Investigación de 2012 del Ministerio de Economía y Competitividad CGJ2012-32914, cofinanciado por Fondos FEDER. 


\begin{tabular}{|c|c|c|}
\hline Caracteres & subsp. muralis & subsp. gaditana \\
\hline \multicolumn{3}{|l|}{ Hojas } \\
\hline dimensiones $(\mathrm{cm})$ & $3,7-21,0 \times 0,7-8,5$ & $3,6-20,5 \times 1,3-6,8$ \\
\hline morfología general & $\begin{array}{c}\text { liradas, lóbulo terminal hastado a } \\
\text { orbicular }\end{array}$ & $\begin{array}{c}\text { liradas, lóbulo terminal orbicular } \\
\text { o deltoide }\end{array}$ \\
\hline morfología hojas superiores & liradas o bracteiformes & oblongas o panduradas \\
\hline margen & angulado & sinuado \\
\hline auriculas & agudas (hasta $0,9 \mathrm{~cm}$ ) & \pm redondeadas (hasta $1,5 \mathrm{~cm}$ ) \\
\hline \multicolumn{3}{|l|}{ Inflorescencias } \\
\hline aspecto sinflorescencia & paniculiforme & corimbiforme \\
\hline involucro (mm) & $5,6-10,2 \times 1,5-2,9$ & $6,9-9,2 \times 1,8-2,7$ \\
\hline \multicolumn{3}{|l|}{ Flores } \\
\hline ligula (mm) & $4,2-6,2 \times 1,0-1,8(2,4)$ & $4,1-5,9 \times 1,0-2,0$ \\
\hline tubo $(\mathrm{mm})$ & $4,6-6,3$ & $3,9-4,6$ \\
\hline estambres (mm) & $1,9-2,5$ & $1,8-2,4$ \\
\hline \multicolumn{3}{|l|}{ Frutos } \\
\hline color & $\begin{array}{l}\text { negro o castaño muy obscuro, pico } \\
\text { color marfil }\end{array}$ & Ocre-pardo, pico concoloro \\
\hline cuerpo del aquenio (mm) & $2,3-3,6 \times 0,6-1,1$ & $2,5-3,4 \times 0,7-1,0$ \\
\hline pico $(\mathrm{mm})$ & $0,5-1,2$ & $0,4-0,5$ \\
\hline vilano (mm) & $4,8-6,4$ & $4,5-5,3$ \\
\hline
\end{tabular}

Tabla 1. Comparación de algunos caracteres morfológicos de interés taxonómico entre Cicerbita muralis (L.) Wallr. subsp. muralis y subsp. gaditana Mejías. Comparison of some morphological characters of taxonomic interest in Cicerbita muralis (L.) Wallr. subsp. muralis and subsp. gaditana Mejías

Se trata de una especie poco variable desde el punto de vista morfológico, a pesar del tamaño de su área de distribución. No obstante, al estudiar el material de la Península Ibérica (véase Apéndice I) con vistas a la síntesis taxonómica para Flora iberica ha puesto de manifiesto que las plantas de la única población conocida de Andalucía Occidental, localizada en la Sierra de Grazalema (Aparicio \& Silvestre 1987), muestran diferencias morfológicas claras respecto al resto de las poblaciones peninsulares. Así, las plantas de todas las poblaciones ibéricas, norteafricanas $\mathrm{y}$ europeas que han sido revisadas presentan aquenios obovados, negros o de un castaño muy oscuro, con pico discoloro de color marfil en la mayor parte de su longitud (figura 1C). Por el contrario, en la población de Grazalema las plantas tienen aquenios de contorno similar pero están menos comprimidos dorsiventralmente, son de color ocre-castaño y muestran un pico concoloro, algo más robusto y más corto (fig. 1B, C). Además, la singularidad de las plantas gaditanas se manifiesta también en otros caracteres, entre los que destacan la morfología foliar y la disposición de los capítulos en una sinflorescencia corimbiforme 


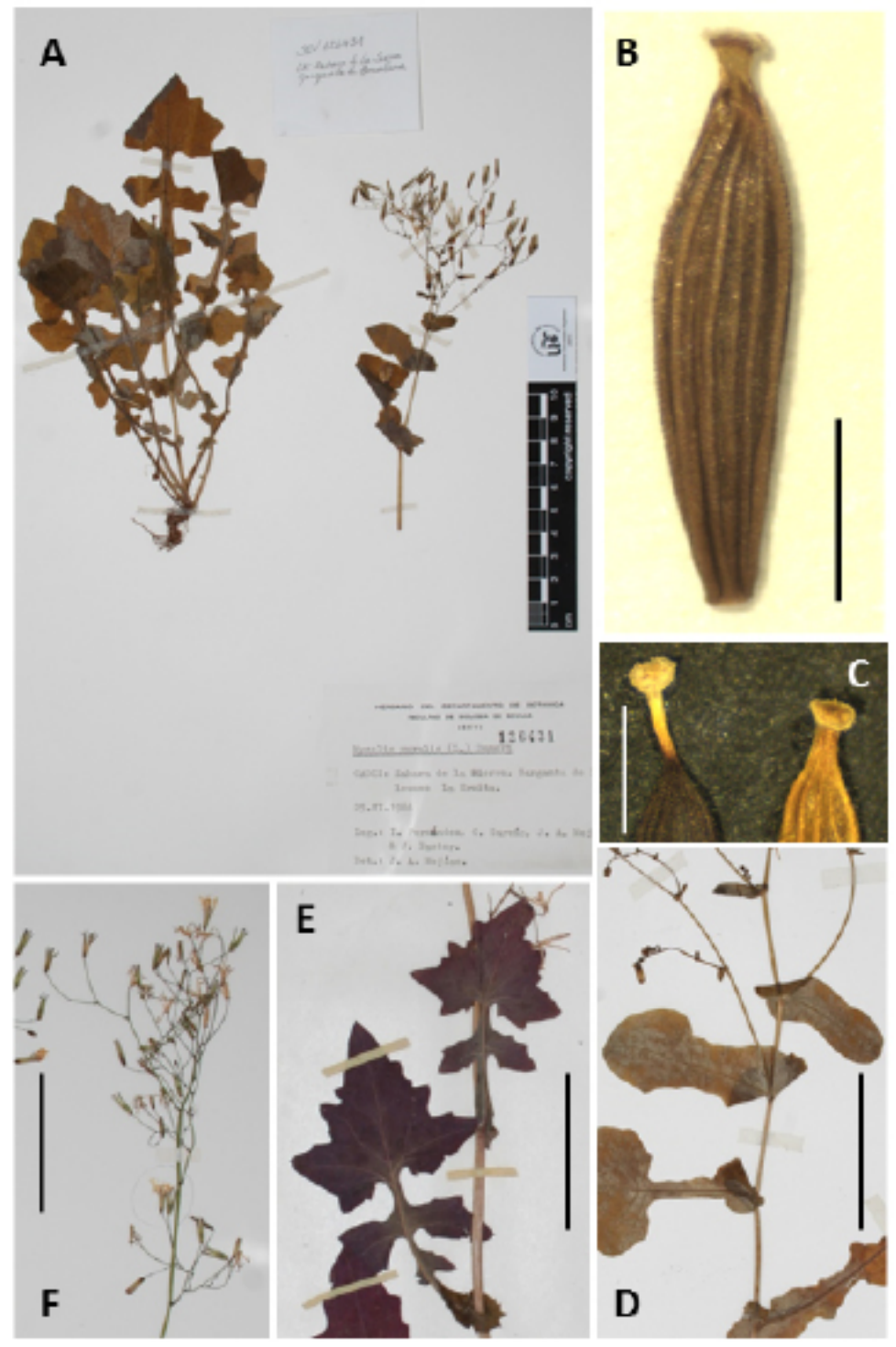

Figura 1. Cicerbita muralis subsp. gaditana: A, holotipo (Hs, Cádiz: Zahara de la Sierra. Garganta de Bocaleones, la Ermita. SEV 126431); B, aquenio maduro; C, detalle del pico del aquenio en C. muralis subsp. muralis (izq., Hs, Gerona: Sant Aniol. SEV 126432) y C. muralis subsp. gaditana (dcha., Hs, Cádiz: Zahara de la Sierra. MA461783); D, hojas caulinares superiores (Hs, Cádiz: Zahara de la Sierra. SEV 110992). Cicerbita muralis subsp. muralis: E, hojas caulinares (Hs, Teruel: Parras de Martín. MA 140242); F, sinflorescencia (Hs, Alava: Pipaón. MA 534095). Escalas: B-C $=1 \mathrm{~mm} . \mathrm{D}-\mathrm{F}=5 \mathrm{~cm}$. Cicerbita muralis subsp. gaditana: A, holotype (Hs, Cádiz: Zahara de la Sierra. Garganta de Bocaleones, la Ermita. SEV 126431); B, mature achene; C, morphological comparison of fruit beak in subsp. muralis (Hs, Gerona: Sant Aniol. SEV 126432, izq.) and subsp. gaditana (Hs, Cádiz: Zahara de la Sierra. MA461783, drch.); D, upper steam leaves (Hs, Cádiz: Zahara de la Sierra. SEV 110992). Cicerbita muralis subsp. muralis: E, upper steam leaves (Hs, Teruel: Parras de Martín. MA 140242); F, synflorescence (Hs, Alava: Pipaón. MA 534095). Scale bars: $B-C=1 \mathrm{~mm} . D-F=5 \mathrm{~cm}$. 
(tab. 1, fig. 1A, D).

La población de la Sierra de Grazalema se localiza a más de $300 \mathrm{~km}$ de distancia de otras más cercanas, en Andalucía oriental y el Sistema Central (en el norte de Extremadura); es, por tanto, una población geográficamente aislada. Los caracteres morfológicos más importantes que diferencian a sus individuos corresponden al fruto y son de gran importancia sistemática en el grupo (Stebbins 1937, Tuisl 1968, Feráková 1977, Koopman et al. 1998), lo que avala el reconocimiento que se hace aquí de ellos como un nuevo taxón con categoría de subespecie, que se describe a continuación.

Cicerbita muralis subsp. gaditana Mejías, subsp. nova

Perennial herbs, rhizomatous, usually single-stemmed, glabrous; rhizome up to $1.2 \times$ $0.7 \mathrm{~cm}$. Stems $25-55 \mathrm{~cm} \times 1.2-2.6 \mathrm{~mm}$, erect, cylindrical in cross-section, unbranched or sparsely branched in the upper part. Basal leaves 9.2-20.5 × 3.1-6.7 cm, lyrate, with \pm winged petiole; caulinar leaves 3.6-14.2 $\times 1.3-6.8 \mathrm{~cm}$, sessile, amplexicaul, with rounded or irregular auricules to $1.5 \mathrm{~cm}$, most of them lyrate, but the upper ones simple, oblong to pandurate. Synflorescence, a corymbose panicle including some tens to hundreds of flower heads, all of them with 5 florets. Involucre 6.9-9.2 × 1.8-2.7 mm with 7-9 bracts in two series, the outer one much smaller. Corolla, yellow; tube 3.9-4.6 mm, glabrous; limbo 4.1-5.9 × 1.0-2.0 mm, villous at the base. Anthers 1.8-2.4 mm, yellow. Style branches, yellow. Achenes 2.9-3.9 x 0.7-1.0 mm, rostrate; body 2.5-3.4 $\mathrm{mm}$, narrowly obovate, \pm compressed, 10- to 13-ribbed longitudinally, scabrescent, ocher colored; peak 0.4-0.5 mm, concolorous; disk c. $0.5 \mathrm{~mm}$, with villousvelutinous margin. Pappus of two series of scabrous bristles, 4.5-5.3 mm, outers slightly shorter than inners, bright white. $2 n=18$.

Ethymology: native from Cádiz province
(Spain).

Holotypus: Hs, Cádiz: Zahara de la Sierra. Garganta de Bocaleones, la Ermita (Spain). 25.VI.1986, I. Fernández, C. García, J. A. Mejías \& J. Pastor (SEV 126431, Mejías I1/86).

Hierba perenne, por lo generalmente unicaule, glabra, rizomatosa, con rizoma hasta de $1,2 \times 0,7 \mathrm{~cm}, \pm$ horizontal y nudoso. Tallos 25-55 cm $\times 1,2-2,6 \mathrm{~mm}$, erectos, pobremente ramificados en el tercio superior, cilíndricos, con costillas longitudinales poco marcadas, a veces casi imperceptibles, fistulosos en la parte inferior, glabros, verdosos. Hojas $(1,8) 3,6-20,5$ $\times 1,3-6,8 \mathrm{~cm}$, alternas, en su mayoría liradas, con 1-3(4) pares de lóbulos laterales hasta de $2,3 \times 1,9 \mathrm{~cm}$, ovados, romboidales $\mathrm{o}$ reniformes, con el lóbulo terminal \pm orbicular, de base cordada o deltoide, glabras, verdes; las superiores simples, panduradas u oblongas, con el margen de entero a sinuado y provisto de pequeños mucrones dispersos; las basales 9,2-20,5 ×3,1-6,7 cm, por lo general en roseta, liradas, con pecíolo \pm alado; las caulinares 3,6-14,2 × 1,3-6,8 cm, las inferiores liradas y las superiores simples y a veces bracteiformes, sésiles, amplexicaules, con aurículas hasta de $1,5 \mathrm{~cm}$, redondeadas o irregulares, nunca agudas. Capítulos numerosos, cada uno con 5 flores liguladas del mismo tamaño, dispuestos en una panícula terminal laxa, corimbiforme, con una hoja lanceolada de 1,5-17,0 $\times$ $1,0-8,5 \mathrm{~mm}$ en cada nudo, pedunculados, con pedúnculos de 1,9-15,3 mm, y 0-2 brácteas de $1,0-1,5 \mathrm{~mm}$, lanceoladas. Involucro $6,9-9,2 \times$ 1,8-2,7 mm, cilíndrico, ligeramente acrescente durante la fructificación, con 2 series de brácteas, la externa mucho más pequeña que la interna; brácteas herbáceas, planas, adpresas, débilmente pubérulas en el ápice, verdosas; las externas 2-3(4), de 0,8-4,8 × (0,4)0,8-1,1 mm, irregularmente dispuestas, lanceoladas, agudas, de margen entero; las internas 5, de 6,7-8,6 
$\times$ 0,9-1,5 mm, linear-lanceoladas, obtusas, de margen escarioso estrecho. Receptáculo \pm $1,7 \mathrm{~mm}$ de anchura, ligeramente convexo en la fructificación, alveolado, glabro. Corola $8,0-10,4 \mathrm{~mm}$, amarilla, vilosa en la base del limbo; tubo 3,9-4,6 mm; limbo 4,1-5,9 × 1,0-2,0 $\mathrm{mm}$. Anteras 1,8-2,4 mm, amarillas. Ramas estilares $\pm 1,2 \mathrm{~mm}$ de longitud, amarillas. Aquenios 2,9-3,9 × 0,7-1,0 mm, rostrados; cuerpo del aquenio 2,5-3,4 mm, estrechamente obovado, \pm comprimido, con 10-13 costillas longitudinales, 5 dorsales más 5-8 ventrales, de márgenes engrosados semejantes a costillas, escábrido, de color ocre; pico 0,4-0,5 mm, concoloro, terminado en un disco de c. $0,5 \mathrm{~mm}$ de diámetro y margen viloso-velutino. Vilano 4,5-5,3 mm, escábrido, con 2 series de pelos, la externa más corta que la interna, de color blanco brillante, prontamente caedizo.

Fenología: florece de mayo a junio (julio).

Número cromosómico: $2 n=18$ (Mejías 1993). Este número somático coincide con el de la subespecie típica y el análisis de la morfología cromosómica muestra gran semejanza con el cariotipo de la misma, con coeficientes de asimetría cromosómica muy similares (Mejías loc.cit.).

Hábitat: herbazales ligeramente nitrófilos, en barrancos y gargantas húmedas y sombrías, en calizas jurásicas; 400-500 msm.

Distribución: Endemismo de la Sierra de Grazalema, Cádiz (España).

Observaciones.- El taxón ha sido recolectado al menos en dos ocasiones durante los años ochenta del siglo pasado (ver Apéndice). Durante ese período presentaba un efectivo de sólo unas decenas de individuos establecidos en un enclave de extensión limitada (observación personal), aunque puedan crecer algunos ejemplares más en algún otro punto de la garganta. La viabilidad de la población, no obstante, está garantizada por la alta autofecundación espontánea y la abundancia de aquenios que producen, pues el nivel de fructificación se acerca al $90 \%$ en años favorables (Mejías 1994).

AGRADECIMIENTOS. Al Herbario de la Universidad de Sevilla (SEV) y a su personal por la ayuda y disponibilidad, en especial a Francisco Javier Salgueiro, quien hizo las fotos de los especímenes. Igualmente, a los herbarios BC, MA, MGC, SALA, SALAF, SANT y VAL, que cedieron material para su estudio. Al Prof. J.A. Devesa, quien ha mejorado de forma significativa la redacción del manuscrito.

\section{APÉNDICE I}

(Material estudiado y revisado)

\section{C. muralis subsp. gaditana}

ESPAÑA. Cádiz: Zahara, Garganta de Bocaleones (TF-85 77), 13.V.1983, Aparicio \& García (MA 461783). Zahara, Garganta de Bocaleones, 13.V.1983, Aparicio et al. (SE V96409). Zahara de la Sierra, Garganta de Bocaleones, La Ermita, 25.VI.1986, Fernández et al. (SE V126431). Zahara, sin fecha ni recolector (SE V110992).

\section{C. muralis subsp. muralis}

ESPAÑA. Álava: Lagrán, sierra de Cantabria, 15.VIII.1992, Alejandre (MA 534083). Maestu, Apellaniz, Los Bardales, 27.VII.1999, UribeEchebarría (MA 642425 \& VAL 42278). Pipaón, sierra de Cantabria, 22.VIII.1992, Alejandre (MA 534095). Salvatierra, Sierra de Bitigarra, 11.IX.1988, Alejandre (MA 486144). Albacete: Fábrica de Riópar, Valle del Chorro, 23.VI.197, Cannon et al. (SEV 52214). Fábricas de Riópar-Villaverde de Guadalimar, Valle del Chorro, nacimiento del río Mundo, 2.VIII.1985, Mejías et al. (SEV 126426). Los Chorros pr. Riópar, 09.VII.1923, Cuatrecases (BC 35873). Los Chorros, Riópar, 20.VII.1934, González-Albo (MA 140229). Los Chorros, Riópar, 
Heywood, sin fecha (MA 183550). Riópar, Los Chorros, 07.VI.1954, Heywood (MA 206081). Riópar, Los Chorros, 30.VI.1957, E.C.C. (SEV 51127). Riópar, Peñas del Gallinero, 23.VII.1983, Herranz (MA 319588). S $S^{\text {a }}$ de Alcaraz, Calar del Mundo, VI.1981, Guerra et al. (MGC 41361). Alicante: Orihuela, montis La Atalaya, 18.VIII.1936, Font Quer (SALA 11497). Asturias: Covadonga, VIII.1966, Segura Zubizarreta (MA 352962). Desfiladero de las Vegas, 14.VIII.1945, Vicioso (MA 140231). Entre Arenas de Cabrales y Camarmeña, 21.IX.1985, García \& Mejías (SEV 126430). Monasterio de Hermo, 14.VIII.1987, Barrera \& Mejías (SEV 126444). Valle de Lago, Somiedo, 27.VIII.1985, Aedo (MA 611518). Barcelona: Ca l'Arenes, Font del Fangar, El Corredor, 06.X.1945, Montserrat (BC 618154). Gualba (Montseny), ex Herb. J.M. Barnades (BC 603842). Les Guilleries: Plá de les Arenes, Herb. Tremols (BC 651181). Montseny, près Sta. Fe, 14.VII.1913, Sennen (BC 875017). Montseny, Rambla de Moscaroles, 09.V.1915, Garriga (BC 35883). Montserrat, VII.1879, Tremols (MA 140244). Montserrat, VIII.1914, Caballero (MA 140245). Riera d'Esparragueres (Sta. Pau, Garrotxa), VIII.1944, Bolós (BC 103193). S. Bartomeu Sesgorgues, 19.VII.1923, Gonzalo (BC 875015). Sot de les Obagues, Montnegre, 03.VII. 1946, Montserrat (BC 618159). Burgos: La Revilla, crestones de la umbría de la Meseta del Carazo, 11.VII.1989, Gil Zúñiga \& Alejandre (MA 484239). San Martín de Humada, Monte Portillo, 29.VII.1987, Fz. de Betoño \& Alejandre (MA 422925). Santo Domingo de Silos, La Yecla, 11.VII.1979, Pons-Sorolla \& Susanna (MA 413072). Sierra de la Demanda, 02.VIII.1977, Fuentes (MA 444537). Sierra de Urbión, Barranco de Río Frío, 6.VIII.1988, Gil Zúñiga \& Alejandre (MA 486143). Cáceres: Hervás, 28.VI.1979, Amich et al. (MA 244720 \& SALA 20433). Hervás, 27.VI.1980, Rico (SALA 48305). Cantabria: Páramo de la Lora (Valderredible), 29.VI.1983, Herrá et al. (MA 685922). Castellón: Bejís, Nacimiento del río Palancia, 23.VI.1987, Burgaz et al. (VAL 8399). Cinctorres (Els Ports), rambla de Cellumbres, La Roca Parda, 02.VIII.1988, Aguilella
\& Renard (VAL 174989 \& VAL 174991). Entre el Mas de la Cambra y La Estrella (Vistabella), 20. VI.1987, Fabregat (VAL 63518). Fredes (El Baix Maestrat), Bc. Dels Avellaners, 22.VI.1988, Aguilella (VAL 179990). Vistabella del Maestrazgo, Cavanilles (MA 140232). Vistabella del Maestrazgo, Molí de 1'Assor, 21.VII.1988, Güemes et al. (VAL14981). Cuenca: Alrededores de las lagunas de El Tobar, 14.VII.1941, Caballero (MA 140239). Alto Tajo, Vega del Codorno, Collado Manchego, 11.VIII.1989, Muñoz et al. (MA 558575). Balneario de Solán de Cabras, 21.VII.1978, López (MA 449108, MA 448087). Ciudad Encantada, Mirador de Uña, 3.VIII.1974, López (MA 444052). Ciudad Encantada, 20.VII.1970, Valdés (SEV 8133). Hoz de Beteta, 09.VII.1932, sin recolector (MA 140240). Mirador de Uña y Ciudad Encantada, 3.VIII.1974, López \& Valdés-Bermejo (MA 444049). Nacimiento del río Cuervo, 31.VII.1985, Rivera \& de la Torre (MA 449771). Gerona: Alt Empordá: Requesens, VII.1879, Tremols (BC 650720). Castanyadell, Costa dels Ases, VII.1868, Masferrer (BC 38894). Entre Campdevanol y Aigües de Ribes, 9.VIII.1985, Mejías et al. (SEV 126438). Garrotxa: Olot, VII.1878, Herb. Tremols (BC 650724). Muntayes de Prades, Vall de Castelfollit, 28.VI.1954, Batalla (BC 598540 \& BC 598541). Muntayes de Prades, Massis de la Mussara, 23.IX.1956, Masclans (BC 598539). Muntayes de Prades, La Vall, 08.VIII.1951, Batalla (BC 598542). Olot, Santa Margarida, 18.VII.1993, Monasterio-H et al. (MA 528802). Rivière d'Arbucias vers Ganduix, 5.IX.1930, Sennen (BC 35865). Sárdenas, ermita de Sant Aniol, 08.VIII.1985, Mejías et al. (SEV 126432). Sárdenas, camino a Sant Aniol, 8.VIII.1985 Mejías et al. (SEV 126435). Rivière d'Arbucias ves Ganduix, 05.IX.1930, Sennen (BC 875016). Torrent de Can Vinyes, 28.VI.1920, sin recolector (BC35866). Guadalajara: Fuente Vivero, refugio hacia Zaorejas, 01.IX.1995, Marín (VAL 96202). Galve de Sorbe, 30.VII.1988, Mateo (VAL 60658). Huesca: Benasque, Plan de Senarta, 9.VIII.1982, Devesa (SEV 78796). Bujaruelo, 25. VII.1982, Boisset (VAL 1281). Candanchú, 05. VIII.1972, Ron (MA 192723 \& SALA 4428). Congosto de Ventamillo, Valle de Benasque, 17. 
VII.1986, Giráldez (MA 393242). Entre Linás de Broto y Yesero, Puerto de Cotefablo, 24.VII.1969, Silvestre \& Valdés (SEV 43526). Ordesa, Torla, camino Cotatuero, 20.VIII.1987, Montserrat et al. (BC 858206). Oto, cerca del Barranco de Yosa, sin fecha, sin recolector (BC 866324). Puerto de Cotefablo entre Linás de Broto y Yesero, 24. VII.1969, Silvestre \& Valdés (SEV 51128). Puerto de Cotefablo, entre Linás de Broto y Yesero, 12.VIII.1985, Mejías et al. (SEV 126436). Sallent de Gállego, Gargantas del río Aguaslimpias, 10. IX.1986, Mejías \& Muñoz (SEV 126443). San Cosme de Guara, VII.1903, Pau (MA 140243). San Juan de la Peña, 22.VII.1976, Cabezudo et al. (MGC 42894). San Juan de la Peña, 22.VII.1976, Casaseca (SALA 10967). Valle de Ansó, entre Ansó y Zuriza, 2.VIII.1978, Devesa et al. (SEV 44486). Valle de Añisclo, 5.VIII.1979, Amich et al. (SALA 22830). Valle de Añisclo, 9.IX.1986, Mejías \& Muñoz (SEV 126442). Valle de Benasque, Congosto Ventamillo, 17.VII.1986, Giráldez (SALA 41795). Jaén: Cazorla, por encima de Fuentes del Guadalquivir, 24.VII.1971, Fernández Casas (MA 415811). Cazorla, Barranco del Guadalentín, 25.IX.1975, González Rebollar et al. (MA 491387). Cazorla, Arroyo de Valdeazores, 17.VII.1984, Fernández (MA 421386). Santiago de la Espada, Barranco del río Borosa, 9.VII.1976, Muñoz et al. (MA 481052). Santiago de la Espada, Barranco del río Madera, Cerro de la Misa, 12.VII.1978, Pajarón \& Rodríguez Pascual (MA 508252). Sierra de Segura, en los roquedos de El Yelmo, 12.IX.1954, Borja et al. (SEV 5491). La Rioja: Entre Villoslada y Achichuelo de Cameros, 13.VIII.1976, Mendiola (MA 504471). Sierra Cebollera, 1.IX.1978, Mendiola (MA 504468). Sierra de la Hez, 13.VIII.1930, Cámara (MA 140230). Sierra de San Lorenzo, Monasterio de Valvanera, 31.VII.1979, Amich (SALA 20856). León: Cordiñanes, 25.VII.1979, Casaseca et al. (SALA 22944). San Pedro de los Montes, 31. VII.1978, Casaseca et al. (MA 476569 \& SALA 47408). Lérida: Cerdanya, Val de Llo, 22.VIII.1916, Sennen (BC 35870). Estany Melreta, pr. Boí, 21.VII. 1944, F.Q. (BC 94449). Inter Caldes de Boí et lacum Estang de Cavallers, 22.VII.1944, F.Q. (BC 94451).
Pallars: Perbes, 07.VIII. 1954, Bolós (BC 486621). Spot, 3.VIII.1982, Fernández Díez (MA 244717 \& SALA 25845). Lugo: Cervantes, Sierra de Ancares, al pie de Tres Obispos, 27.VII.1986, Nieto Feliner \& Pedrol (MA 440767). Devesa de Rogueira, 22. VII.1980, Izco et al. (SANT 15839). Esperante, c. del puente sobre el Lor, 24.VI.1981, Izco et al. (SANT 14875). Triacastela, devesa del Oribio, 13. VIII.1994, Amigo \& Romero (SANT 28180). Madrid: Miraflores de la Sierra, bajando el puerto de Canencia hacia Canencia, 29.IX.1988, Izuzquiza et al. (MA 452083 \& MA 499671). Somosierra, 23.VII.1858, Isern (MA 140237). Navarra: Aranaz, San Juan Xarr, 08.VII.1983, Aizpuru \& Catalán (MA 716685). Arce, Peña Ponsoroa, Aizpuru \& Catalán (MA 716683). Contrasario, La Cuestión, 1.VII.1989, Casaseca et al. (SALA 95442). Guesalaz, montes de Iturgoyen, Mugaga, 24.VI.1989, Alejandre (MA 484226). Isaba, 25.VI.1977, Amich et al. (SALA 20354). Navascués, Alto del Borreguil, 03.VIII.1987, Aizpuru \& Catalán (MA 716684). Yerri, sierra de Urbasa, 23.VII.1989, Gil Zúñiga \& Alejandre (MA 484238). Orense: Carballeda, Teixadal de Peña Trevinca, 19.VII.1984, Ortiz (SANT 16673). Palencia: Dehesa de Montejo, valle de Tosande, 14.VII.1995, Monasterio-H. et al. (MA 560617). Salamanca: Castañares de las Honfrías, Linares de Riofrío, 15.VI.1974, Castroviejo (MA 593630). Dehesa de Candelario, Candelario, 26.VII.1982, Navarro \& Valle (SALAF 4787). Las Honfrías, Linares de Riofrío, 31.VII.1973, Fernández Díez (SALA 5557). Linares de Riofrío, 08.VII.1979, Fernández Alonso (MA 519069). Linares de Riofrío, La Honfría, 18.VI.1987, Amor (SALA F16029). Montemayor del Río, 28.VI.1984, Rico et al. (SALA 36877). Segovia: Aldealengua de Pedraza (puerto de Lozoya), 9.VII.1982, Romero (MA 567918). Aldealengua de Pedraza, 12.VII.1987, Egido \& García (MA 743480). Aldealengua de Pedraza, 17.VII.1985, García (MA 743479). Arcones (La Dehesa), 19.VI.1983, Romero (MA 568441). Cerezo de Arriba, cabecera del río San Benito, 21.VII.1983, Romero (SALA 39639). Navafría, 08.VII.1979, Silvestre (MA 222178 \& SEV 57605). Revenga, 22.VII.1991, Martínez García (MA 696558). San 
Ildefonso, Valsaín, 19.VII.2009, Calvo \& Hantson (MA 789757). Valsaín, 04.VIII.1990, Egido \& García Adá (MA 743478). Soria: Covaleda, 26.VIII.1959, Segura Zubizarreta (MA 352997). Subida al Puerto de Santa Inés, 18.IX.1985, García \& Mejías (SEV 126440). Tarragona: Cardó, Barranco del Domenge, 03.VIII.1972, Toldi (BC 628620). Fortem “el Pastor" (Cardó), 29.VI.1942, Font Quer (BC 93490). La Sénia, El Retaule, 27.VII. 1988, Aguilella et al. (VAL 174992). Mas de Barberans, 2.VII.1983, Mateo \& Aguilera (MA 442931). Obaga de la Sierra de Formigosa, 28.IX.1997, Mayol \& Sáez (MA 597715). Pista de Fredes al monte Caro, Barranco de Rafalgari, 8.VI.1999, Navarro et al. (MA 626735). Ports de Tortosa, 27.VI.1917, Font Quer (BC 35880). Sierra de Montsiá, San Carles de la Rápita, Font de Burgá, 11.VI.1999, Velayos et al. (MA 626406). Teruel: Beceite, pr. El Parrizal, 27.V.1989, Mateo (VAL 64556). Cañón del Guadalope, pr. Aliaga, 17.VII.1988, Mateo (VAL 882736). El Parrizal, Beceite, 17.VII.1982, Rico \& Sánchez (SALA 32120). Ermita del Tremedal, 24.VII.1988, Mateo (VAL 59826). Noguera de Albarracín, Barranco Nabellida, 29.VI.2006, Fabado et al. (VAL 161180, VAL 182681). Parras de Martín, 27.VII, Seal (Pau)? (MA 140242). Tramacastilla, Almagro, sin fecha (VAL 155068). Villarluengo, Salto de la Zorra, 10.VII.1993, Mercadal (VAL 81706). Valencia: Barranco de la Hoz, pr. Puebla de San Miguel, 20.VII.1988, Mateo (VAL 882490). Vallanca, pie de cantiles húmedos y sombreados, 12.VI.1985, Mateo \& Figuerola (MA 313510). Vallanca, pie de cantiles húmedos y sombreados, 12.VI.1985, Mateo \& Figuerola (VAL 51725). Vizcaya: Ichine, Gorbea, 24.VII.1948, Guinea (MA 244155). Peñascal grande de Iturrigorri, 10.VII.1946, Guinea (MA 154708). Zamora: Ribadelago, 17.VIII.1987, García \& Roa (MA 510615).

ALEMANIA. Schleswig-Holstein: Norder Gosharde, N of Bredstedt, 07.09.1978, Larsen \& Larsen (SEV 116190).

ANDORRA. Ordino, Sispony, subida al Pic de Carroi, 1560 msm, 28.VIII.2002, Aedo et al.
(MA 700274).

FRANCIA. Hautes-Pyrénées: Valle del río Aure. Subida a Cap de Long, 8.IX.1986, Mejías \& Muñoz (SEV 126441).

INGLATERRA. Gloucester: Leckhampton, 21.VIII.1970 (MA 724675).

ITALIA. Lazio: Tivoli, Villa d'Este, 06.VIII.2014, Mejías (SEV 286481). Sicilia: Nebrodi, Portella Femmina Morta, 1500-1600 m, 09.06.1990, Raimondo et al. (SEV 263257).

MARRUECOS. Al-Hoceima: Azila, Koudinat Tighighine, Jbel Imaou Chabane, $1650 \mathrm{~m}$, 30.06.1993, Mejías \& Silvestre (SEV 139081). Cerca de Ketama, subiendo al monte Koudiet Imoûgrâs, 1880 msm, 22.VI.1982, Fdez. Casas et al. (MA 244719). Beni-Hasan: Tamalot, 950 m, 19.VI.1928, Font Quer (MA 140219). Rif SW: Massif calcaire central, vallon de Tasnoute, 08.VI.1955, Sauvage (MA 168091).

SUIZA. Canton de Genève : environs de Genève: au Coin, VII.1868, Herbier Henri Bernet (SANT 07922).

\section{BIBLIOGRAFÍA}

APARICIO, A. \& S. SILVESTRE -1987- Flora del Parque Natural de la Sierra de Grazalema. Junta de Andalucía y Agencia de Medio Ambiente. Sevilla.

CHAUVET S., M. VAN DER VELDE, E. IMBERT, M.L. GUILLEMIN, M. MAYOL, M. RIBA, M.J. SMULDERS, B. VOSMAN, L. ERICSON, R. BIJLSMA \& B. E. GILES -2004- Past and current gene flow in the selfing, wind-dispersed species Mycelis muralis in western Europe. Mol. Ecol. 13: 1391-1407.

CZARNECKA, B. -1986- Ecodiagrams of common species of the forest herb layer in the Roztocze National Park. Acta Soc. Bot. Poloniae 55: 429-466. 
CLABBY, G. \& B. A. OSBORNE -1999- Biological flora of the British Isles: Mycelis muralis (L.) Dumort. (Lactuca muralis (L.) Gaetner. J. Ecol. 87: 156-172.

DEVESA, J. A. \& S. TALAVERA -2014- in J. A. DEVESA, A. QUINTANAR \& M. Á. GARCÍA (eds.), Flora iberica, XVI (I): 5-22. Real Jardín Botánico, C.S.I.C. Madrid.

FERÁKOVÁ, V. -1976- in T. G. Tutin et al. (eds.), Flora Europaea 5: 331-332.

FERÁKOVÁ, V. -1977- The genus Lactuca L. in Europe. Univerzita Komenského, Bratislava.

HULTÉN, E. \& M. FRIES -1986- Atlas of north european vascular plants, north of the tropic of Cancer. Koeltz Scientific Books, Königstein.

KILIAN, N., B. GEMEINHOLZER \& H. W. LACK -2009- in V. A. Funk, A. Susanna, T. F. Stuessy \& R. J. Bayer (eds.): Systematics, evolution and biogeography of Compositae: 343-383. International Association for Plant Taxonomy, Viena.

KOOPMAN, W. J. W. -2005- Phylogenetic signal in AFLP data sets. Syst. Biol. 54: 197-217 (2005).

KOOPMAN, W. J. M., E. GUETTA, C. C. M. VAN DE WIEL, B. VOSMAN \& R. G. VAN DEN BERG -1998- Phylogenetic relationships among Lactuca (Asteraceae) species and related genera based on ITS-1 DNA sequences. Amer. J. Bot. 85: 1517-1530.

KOOPMAN, W.J.M., M.J.ZEVENBERGEN \& R.G. VAN DEN BERG -2001- Species relationships in Lactuca s.1. (Lactuceae, Asteraceae) inferred from AFLP fingerprints. Amer. J. Bot. 88: 1881-1887.

MEJÍAS, J. A. -1993- Mediterranean chromosome number reports: Prenanthes, Cicerbita and Mycelis in the Iberian Peninsula. Fl. Medit. 3: 333-338.

MEJÍAS, J. A. -1994- Self-fertility and associated flower head traits in the Iberian taxa of Lactuca and related genera (Asteraceae: Lactuceae). Pl. Syst. Evol. 191: 147-160.
PRESTON, CH. D. \& M. O. HILL -1997- The geographical relationships of Britain and Irish vascular plants. Bot. J. Linn. Soc. 124: 1-120.

STEBBINS, G. L. -1937- Critical Notes on Lactuca and related genera. J. Bot. 75: 12-18.

TUISL, G. -1968- Der Verwandtschaftskreis der Gattung Lactuca L. in iranischen Hochland und seinen Randgebieten. Ann. Naturhistor. Mus. Wien, $B$ 72: 587-638.

WANG, Z. H., H. PENG \& N. KILIAN -2013Molecular Phylogeny of the Lactuca Alliance (Cichorieae Subtribe Lactucinae, Asteraceae) with Focus on Their Chinese Centre of Diversity Detects Potential Events of Reticulation and Chloroplast Capture. Plos One 8: e82692.

Dirección del autor: Departamento de Biología Vegetal y Ecología, Universidad de Sevilla. Avda. Reina Mercedes, 6. Apartado 1095, 41080 Sevilla (Spain).E-mail: jmejias@us.es. 